\title{
Motivos para prática de esporte em idades jovens: Um estudo de revisão
}

\author{
Sport practice motives in young ages: A review study \\ Andrea Gomes Bernardes ${ }^{1}$, Bruna Hatsue Santos Yamaji1, Dartagnan Pinto Guedes ${ }^{1 *}$ \\ ARTIGO DE REVISÃO | REVIEW ARTICLE
}

\begin{abstract}
O objetivo do estudo foi realizar uma revisão da literatura sobre motivos para prática de esporte em idades jovens. A busca de publicações foi realizada nas bases de dados Embase, Medline, PsycINFO e SPORTDiscus, considerando artigos publicados entre 1980 e 2013 nos idiomas português, inglês e espanhol. Na estratégia de busca foram utilizados os descritores "motivo", "motivação", "adesão", "esporte" e "treino" com os limites da pesquisa "criança", "adolescente" e "jovem". Também foram realizadas buscas manuais nas referências dos artigos selecionados. A busca bibliográfica resultou na localização de aproximadamente oito mil artigos. Contudo, 26 preencheram os critérios para compor a revisão. Os resultados demonstraram que os motivos para a prática de esporte em jovens-atletas resultam da interação entre diversos fatores individuais, sociais e ambientais. Os estudos selecionados para analise, em sua maioria, identificaram principalmente motivos vinculados à auto-realização associada ao domínio/aperfeiçoamento de habilidades esportivas, em que a Competência Técnica foi o fator mais apontado pelos jovens-atletas de ambos os sexos inseridos nos diferentes contextos socioculturais.
\end{abstract}

Palavras-chave: motivação, adesão, criança, adolescente, treino

\begin{abstract}
The present study aimed to carry out a literature review of sports practice motives in young ages. The search was conducted in the Embase, Medline, PsycINFOe SPORTDiscus databases, considering articles published between 1980 and 2013 in Portuguese, English and Spanish. In the search strategy were used the descriptors "motive", "motivation", "adherence", "sport" e "training" with the limits of research "child", "adolescent" and "young". Manual searches were also conducted in the references of selected articles. The bibliographic research resulted in locating approximately eight thousand articles. However, 26 met the criteria for compose the review. The results showed that sports practice motives in young ages were identified by interactions between individual, social and environmental factors. Mostly of the studies selected for analysis revealed motives mainly related to self-realizations associated with the domain/improving sports skills in which the Technical Competence was the factor pointed by most athletes-young of both sexes entered in different sociocultural contexts.
\end{abstract}

Keywords: motivation, adherence, child, adolescent, training

\footnotetext{
Artigo recebido a 18.10.2013; Aceite a 22.05.2014

${ }^{1}$ Centro de Pesquisa em Ciências da Saúde, Universidade Norte do Paraná, Paraná, Brasil

* Autor correspondente: Rua Ildefonso Werner, 177, Condomínio Royal Golf, CEP: 86055-545 - Londrina, Paraná, Brasil E-mail: darta@sercomtel.com.br
} 


\section{INTRODUÇÃO}

Informações disponibilizadas na literatura têm destacado os múltiplos benefícios para um adequado desenvolvido físico, mental e social associados à prática de esporte em idades jovens (Boreham, Twisk, Savage, Cran, \& Strain, 1997; Harrison \& Narayan, 2003; Kawabe et al., 2000; Pate, Trost, Levin, \& Dowda, 2000). Porém, contraditoriamente, levantamentos recentes têm apontado que somente uma pequena parcela da população infantil e juvenil pratica esporte com alguma regularidade (Capranica \& Millard-Stafford, 2011; Delorme, Chalabaev, \& Raspaud, 2011; Jõesaar \& Hein, 2011) e, entre aqueles que iniciam a prática esportiva, chama atenção o elevado índice de abandono, com forte repercussão para vida presente e futura dos jovens (Sirard, Pfeiffer, \& Pate, 2006).

Estudos na área demonstram que a motivação pode se constituir em importante determinante para iniciar a pratica de esporte, justificar sua permanência e minimizar os casos de abandono (Capranica \& Millard-Stafford, 2011; Delorme et al., 2011; Sirard et al., 2006). Neste particular, motivos que possivelmente possam estar relacionados à prática de esporte são bastante diversificados de acordo com expectativas estabelecidas pelos próprios praticantes, resultando da interação entre múltiplos atributos socioculturais e ambientais, como por exemplo, em um extremo, motivos associados à diversão e ao convívio de grupos, e em outro extremo, motivos associados ao aprimoramento de aptidão física e à competência técnica (Gill \& Williams, 2008). Neste caso, pressupõe que esses motivos deverão agir diferentemente em cada estágio do processo de desenvolvimento individual dos jovens (Weinberg et al., 2000).

Portanto, a identificação dos motivos subjacentes à prática de esporte possibilita que sejam delineadas ações mais eficientes de incentivo para início de sua prática e que possa levar os jovens a alcançarem em sua maior plenitude as metas propostas, promovendo, desse modo, clima motivacional favorável, o que aumenta as chances de adesão. Diante disso, estudiosos da área vêm procurando aplicar diferentes teorias elaboradas no campo da psicologia que tentam explicar a adesão na prática de esporte (Roberts \& Treasure, 2012).

Considerando que a importância de identificar, dimensionar e ordenar os motivos para a prática de esporte em idades jovens justifica-se com base no pressuposto de que a infância e a adolescência são períodos críticos para iniciar a participação efetiva em programas regulares de esporte ou, pelo contrário, abandonar por completo sua prática, o objetivo do presente estudo foi realizar uma revisão dos achados até então disponibilizados na literatura relacionados aos motivos associados à prática de esporte em idades jovens.

\section{MÉTODO}

$O$ rastreamento dos artigos incluiu pesquisa nas bases de dados eletrônicas Embase, Medline, PsycINFO e SPORTDiscus, no período entre janeiro de 1980 e março de 2013, independente do país de publicação. A estratégia de busca baseou-se nos descritores "motivo", "motivação" e "adesão" combinados com "esporte" e "treino", com os limites da pesquisa "criança", "adolescente" e "jovem", e suas traduções para os idiomas inglês e espanhol. As referências bibliográficas dos estudos assim localizados foram também rastreadas para localizar outras intervenções de potencial interesse e que preenchiam os critérios de seleção para o estudo.

A seleção dos artigos acompanhou procedimento proposto para levantamento de informações disponibilizadas na literatura (Higgins \& Green, 2008) e baseou-se na conformidade dos limites dos descritores, tendo sido desconsiderados aqueles que, apesar de aparecerem no resultado da busca, não abordavam o tema sob o ponto de vista de identificação dos motivos para prática de esporte em idades jovens.

$\mathrm{Na}$ Figura 1 encontra-se o processo usado para rastrear e selecionar os artigos da revisão retrospetiva. No levantamento bibliográfico inicial foram encontradas por volta de oito mil publicações. Excluídas as publicações cruzadas redundantes, constantes em mais de uma base de 
dados, em um segundo momento foram consideradas 4875 publicações, das quais, após atendimento de uma sequência de critérios de seleção, 26 foram consideradas para estudo. Os critérios de seleção considerados foram: (a) estudos publicados em português, inglês ou espanhol; (b) artigos originais; (c) estratificação por idade $\leq 20$ anos; (d) características e seleção da amostra claramente definidas; e (e) identificação dos motivos para prática de esporte mediante instrumento validado. A análise e a seleção dos artigos foram realizadas por dois investigadores sob a supervisão de um terceiro investigador, que reanalisou as publicações excluídas. Eventuais discrepâncias relacionadas às exclusões foram solucionadas por consenso dos três investigadores.

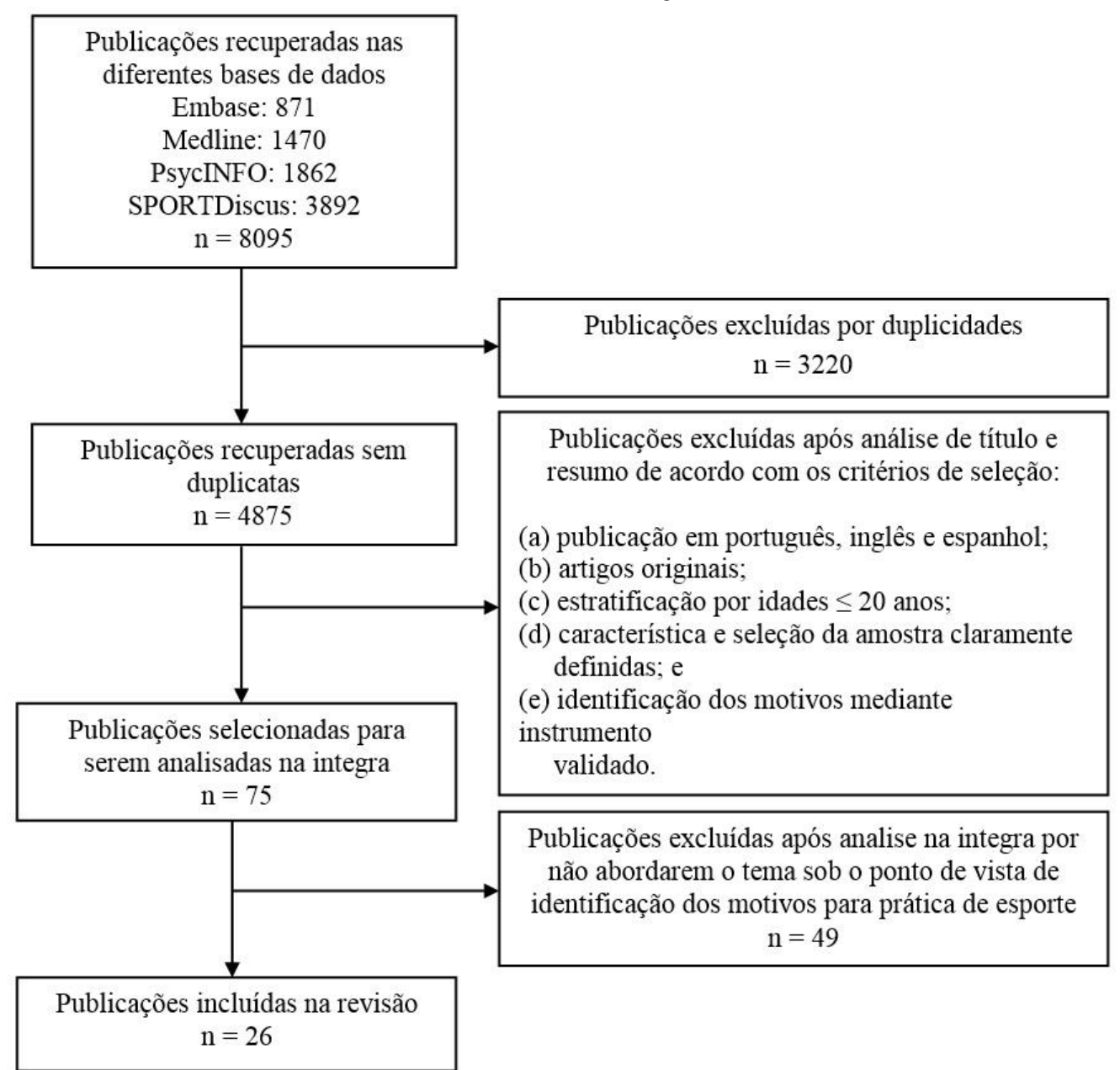

Figura 1. Fluxograma de seleção das publicações para revisão.

\section{RESULTADOS}

De acordo com Quadro 1, verifica-se que os artigos incluídos na revisão foram desenvolvidos em diferentes regiões do mundo, com destaque para aqueles publicados no Brasil que, isoladamente, representa $42 \%$ dos artigos localizados, ou seja, proporção superior de artigos selecionados que foram publicados nos países europeus (31\%). Artigos selecionados e que foram publicados nos Estados Unidos e em todos os demais países representam, respectivamente, $12 \%$ e $15 \%$. Quanto ao ano de publicação, a totalidade dos artigos foi publicada no período de 1983 a 2013, em maior proporção a partir do ano de 2001 (80,8\%). 
166 | AG Bernardes, BHS Yamaji, DP Guedes

Tabela1

Características dos estudos que procuram descrever os motivos para a prática de esporte em jovens-atletas

\begin{tabular}{|c|c|c|c|c|c|c|c|}
\hline Referência & Origem & $\begin{array}{l}\text { Instrumento } \\
\text { de Medida }\end{array}$ & Amostra & $\begin{array}{l}\text { Idade } \\
\text { (anos) }\end{array}$ & Modalidades & $\begin{array}{l}\text { Âmbito de } \\
\text { Prática }\end{array}$ & Motivos \\
\hline Gill et al. (1983) & Estados Unidos & PMQ & $\begin{array}{l}\sigma^{n}=720 \\
\stackrel{9}{=}=418\end{array}$ & $8-18$ & Coletivas e Individuais & Escolar & ơ e $q$ : Competência Técnica. \\
\hline $\begin{array}{l}\text { Longhurst e Spink } \\
\text { (1987) }\end{array}$ & Austrália & PMQ & $\begin{array}{l}\sigma^{x}=187 \\
\stackrel{\uparrow}{=} 217\end{array}$ & $8-18$ & $\begin{array}{c}\text { Atletismo, Natação, } \\
\text { Netball, Cricket e } \\
\text { Futebol } \\
\end{array}$ & $\begin{array}{l}\text { Clubes } \\
\text { esportivos }\end{array}$ & $\begin{array}{l}o^{\pi} \text { e } \text { \%: Competência Técnica. } \\
\text { Diferenças relativas às idades. }\end{array}$ \\
\hline $\begin{array}{l}\text { Curry e Weiss } \\
(1989)\end{array}$ & $\begin{array}{l}\text { Estados Unidos } \\
\text { e Áustria }\end{array}$ & RP-Sport & $\begin{array}{c}\text { Estados Unidos: } \\
\left(\sigma^{\top}=219 \%=178\right) \\
\text { Áustria: } \\
\left(\sigma^{\top}=159 \%=142\right)\end{array}$ & $18-19$ & Coletivas e Individuais & $\begin{array}{l}\text { Universitários e } \\
\text { Clubes } \\
\text { esportivos }\end{array}$ & $\begin{array}{l}\text { Estados Unidos ơ e } q \text { : Competição. } \\
\text { Áustria ơ e } \$ \text { : Aptidão física. }\end{array}$ \\
\hline $\begin{array}{l}\text { Buonamano et al. } \\
\text { (1995) }\end{array}$ & Itália & PMQ & $\begin{array}{l}\bigcirc^{\top}=1411 \\
\wp=1177\end{array}$ & $9-18$ & Coletivas e Individuais & $\begin{array}{l}\text { Iniciantes } \\
\text { Médio } \\
\text { Avançado } \\
\end{array}$ & $\begin{array}{l}\text { ơ e } 9 \text { : Diversão. } \\
\text { Diferenças relativas às idades. }\end{array}$ \\
\hline Kirkby et al. (1999) & $\begin{array}{l}\text { Austrália e } \\
\text { China }\end{array}$ & PMQ & $\begin{array}{c}\text { Austrália } \\
\left(o^{\star} \text { e } \$=280\right) \\
\text { China } \\
\left(o^{\top} \text { e } \$=103\right)\end{array}$ & $8-15$ & Ginástica Artística & $\begin{array}{c}\text { Escolar e } \\
\text { Clube esportivo }\end{array}$ & $\begin{array}{c}\text { Austrália ơ e } \text { o: Diversão e Competição. } \\
\text { China } o^{7} \text { e } \text { o: Afiliação e } \\
\text { Reconhecimento Social. } \\
\text { Diferenças relativas às idades. }\end{array}$ \\
\hline Paim (2001) & Brasil & PMQ & $\begin{array}{l}\sigma^{n}=16 \\
\stackrel{9}{=}=16\end{array}$ & $10-16$ & Futebol & Escolar & $\begin{array}{l}\sigma^{n} \text { e } q: \text { Competência técnica. } \\
\text { Diferenças relativas à idade. }\end{array}$ \\
\hline $\begin{array}{lll}\begin{array}{l}\text { Cecchini } \\
(2002)\end{array} & \text { et } & \text { al. } \\
\end{array}$ & Espanha & $\begin{array}{l}\text { Questionário } \\
\text { Próprio } \\
\end{array}$ & $\begin{array}{l}\sigma^{n}=2294 \\
\stackrel{9}{=} 2312\end{array}$ & $8-18$ & Coletivas e Individuais & Escolar & $\begin{array}{c}\text { o: Saúde e Aptidão Física. } \\
\text { o: Saúde e Competência Técnica. }\end{array}$ \\
\hline Allen (2003) & Nova Zelândia & SMOSS & $\stackrel{q}{q}=100$ & $14-17$ & Coletivas & $\begin{array}{c}\text { Experiência em } \\
\text { esportes }\end{array}$ & \$: Diversão e Afiliação. \\
\hline $\begin{array}{lll}\text { Paim } & \text { e } & \text { Pereira } \\
(2004) & & \\
\end{array}$ & Brasil & IMPD & $\sigma^{n}=18$ & $11-14$ & Capoeira & Escolar & o: Saúde e Afiliação. \\
\hline $\begin{array}{l}\text { Salgueiro et al. } \\
(2004)\end{array}$ & Espanha & PMQ & $\begin{array}{l}\sigma^{n}=204 \\
q=224\end{array}$ & $8-20$ & Natação & $\begin{array}{c}\text { Local } \\
\text { Regional } \\
\text { Nacional } \\
\end{array}$ & $\begin{array}{c}\text { ơ: Reconhecimento Social. } \\
\text { 9: Diversão e Afiliação. } \\
\text { Diferenças relativas às idades. }\end{array}$ \\
\hline Garcia et al. (2005) & Brasil & PMQ & $\sigma^{x}=171$ & $8-14$ & Basquetebol & Iniciantes & $\begin{array}{c}\text { ơ: Diversão. } \\
\text { Diferenças relativas às idades. }\end{array}$ \\
\hline Sit e Lindner (2006) & Hong Kong & PMQ & $\begin{array}{l}\sigma^{\pi}=742 \\
\wp=493\end{array}$ & $14-20$ & Coletivas e Individuais & $\begin{array}{l}\text { Competição } \\
\text { Recreação }\end{array}$ & $\begin{array}{l}\text { o: Diversão e Reconhecimento Social. } \\
\text { ९: Diversão e Afiliação. }\end{array}$ \\
\hline Sirard et al. (2006) & Estados Unidos & PMQ & $\begin{array}{l}0^{\pi}=853 \\
\stackrel{q}{=}=839\end{array}$ & $10-15$ & Coletivas e Individuais & $\begin{array}{c}\text { Local } \\
\text { Regional }\end{array}$ & $\begin{array}{c}\text { o: Competição. } \\
\text { \%: Afiliação e Competência Técnica. }\end{array}$ \\
\hline
\end{tabular}


Motivos para prática de esporte em jovens | 167

\begin{tabular}{|c|c|c|c|c|c|c|c|}
\hline Gurbuz et al. (2007) & Turquia & PMQ & $\sigma^{x}=304$ & $9-15$ & Futebol & Escolar & o: Competência Técnica. \\
\hline $\begin{array}{l}\text { Lopes e Nunomura } \\
(2007)\end{array}$ & Brasil & $\begin{array}{l}\text { Entrevista } \\
\text { Semi- } \\
\text { estruturada }\end{array}$ & $q=20$ & $11-14$ & Ginástica & Competição & $\begin{array}{c}\text { 55\% Prazer pela pratica. } \\
\text { 20\% Necessidades fisiológica e } \\
\text { psicológica. } \\
\text { 10\% Preencher tempo livre. }\end{array}$ \\
\hline $\begin{array}{l}\text { Salselas et al. } \\
(2007)\end{array}$ & Portugal & PMQ & $\begin{array}{l}\sigma^{x}=68 \\
\subsetneq=87\end{array}$ & $7-18$ & Natação & $\begin{array}{c}\text { Iniciante } \\
\text { Avançado } \\
\text { Competição }\end{array}$ & $\begin{array}{c}\text { \%: Afiliação. } \\
\text { Diferenças relativas às idades e } \\
\text { experiência de prática. }\end{array}$ \\
\hline $\begin{array}{l}\text { Interdonato et al. } \\
(2008)\end{array}$ & Brasil & PMQ & $\begin{array}{l}\sigma^{x}=72 \\
q=15\end{array}$ & $12-14$ & Coletivas e Individuais & $\begin{array}{c}\text { Clubes } \\
\text { esportivos }\end{array}$ & ơ e o: Saúde. \\
\hline $\begin{array}{l}\text { Martinez et al. } \\
(2008)\end{array}$ & Espanha & PMQ & $\sigma^{x}=734$ & $8-16$ & Futebol & $\begin{array}{l}\text { Pré-mirim, } \\
\text { mirim, infantil, } \\
\text { infanto-juvenil, } \\
\text { juvenil, júnior }\end{array}$ & $\begin{array}{l}\text { o: Amizade e Diversão. } \\
\text { Diferenças referentes às } \\
\text { idades/categorias. }\end{array}$ \\
\hline Nunez et al. (2008) & Brasil & IMPD & $\sigma^{x}=100$ & $13-16$ & Futsal & Escolar & o: Rendimento esportivo. \\
\hline Zanetti et al. (2008) & Brasil & $\begin{array}{l}\text { Questionário } \\
\text { Estruturado }\end{array}$ & $\begin{array}{l}\sigma^{\pi}=18 \\
\wp=12\end{array}$ & $12-19$ & Voleibol & Regional & $\begin{array}{c}28,9 \% \text { Prazer. } \\
\text { 13,3\% Influencia Familiar. } \\
11,1 \% \text { Ser um grande atleta. } \\
8,9 \% \text { Aprender a trabalhar em equipe. }\end{array}$ \\
\hline $\begin{array}{l}\text { Balbinotti et al. } \\
(2009)\end{array}$ & Brasil & Imprafe-54 & $\begin{array}{l}\sigma^{x}=276 \\
\wp=165\end{array}$ & $13-16$ & Basquetebol & $\begin{array}{c}\text { Municipal } \\
\text { Estadual } \\
\text { Nacional } \\
\end{array}$ & $\begin{array}{l}\text { o: Prazer e Competição. } \\
\text { ९: Prazer e Afiliação. }\end{array}$ \\
\hline $\begin{array}{l}\text { Campos et al. } \\
(2011)\end{array}$ & Brasil & PMQ & $\begin{array}{l}\sigma^{2}=50 \\
\stackrel{9}{=}=46\end{array}$ & $13-20$ & Voleibol & $\begin{array}{c}\text { Atletas } \\
\text { Federados } \\
\text { (mirim, infantil } \\
\text { e juvenil) } \\
\end{array}$ & $\begin{array}{l}\text { ơ e } 9: \text { Competência Técnica. } \\
\text { Diferenças relativas às idades/categorias. }\end{array}$ \\
\hline $\begin{array}{ll}\text { Garyfallos } & \text { e } \\
\text { Asterios (2011) }\end{array}$ & Chipre & PMQ & $\sigma^{x}$ e $\stackrel{\varphi}{=} 152$ & $10-12$ & $\begin{array}{c}\text { Futebol, Basquetebol e } \\
\text { Voleibol }\end{array}$ & Escolar & ơ e ?: Diversão e Afiliação. \\
\hline $\begin{array}{lll}\begin{array}{l}\text { Balbinotti et } \\
(2012)\end{array} & & \text { al. } \\
\end{array}$ & Brasil & Imprafe-126 & $\begin{array}{l}0^{x}=166 \\
q=60\end{array}$ & $13-16$ & Tênis de Campo & $\begin{array}{l}\text { Estadual } \\
\text { Nacional } \\
\end{array}$ & ơ e \%: Prazer. \\
\hline $\begin{array}{l}\text { Januário et al. } \\
(2012)\end{array}$ & Portugal & PMQ & $\begin{array}{l}\sigma^{x}=577 \\
\wp=439\end{array}$ & $10-20$ & Coletivas e Individuais & Escolar & $\begin{array}{c}\text { ơ: Competência Técnica/Aptidão Física. } \\
\text { o: Competência Técnica/Aptidão Física e } \\
\text { Atividade de Grupo. } \\
\text { Diferenças relativas às idades. }\end{array}$ \\
\hline $\begin{array}{l}\text { Guedes e Netto } \\
(2013)\end{array}$ & Brasil & PMQ & $\begin{array}{l}\sigma^{x}=803 \\
\wp=714\end{array}$ & $12-18$ & Coletivas e Individuais & $\begin{array}{l}\text { Regional } \\
\text { Estadual }\end{array}$ & $\begin{array}{c}\text { o: Competição e Competência Técnica. } \\
\text { ९: Atividade de Grupo e Afiliação. } \\
\text { Diferenças relativas à idade. }\end{array}$ \\
\hline
\end{tabular}


Embora tenha sido verificada alguma diversidade no emprego dos questionários de motivação nos estudos selecionados, pode-se destacar predomínio quanto ao uso do Participation Motivation Questionnaire - PMQ (65\%). Com referência às modalidades esportivas, destacaram-se os esportes coletivos voleibol, futebol, basquetebol, além de esportes individuais incluindo natação, tênis de campo e ginástica.

Os resultados indicaram que os motivos para prática de esporte podem se diferenciar de acordo com sexo, idade, modalidade esportiva, tempo de prática e experiência de competição. Neste caso, independentemente da idade e do esporte praticado, em ambos os sexos, verificou-se tendência bastante bem definida para motivos caracterizados pela Competência Técnica, Diversão, Prazer e Saúde. Contudo, se entre as moças, os motivos associados à Afiliação e à Diversão receberam destaque importante, no caso dos rapazes, foram os motivos equivalentes à Competição, ao Reconhecimento Social e à Aptidão física que justificaram mais intensamente a prática de esporte.

\section{DISCUSSÃO}

Os motivos para a prática de esporte em jovens-atletas resultam da combinação de indicadores sociais, ambientais e individuais que determinam a seleção de modalidades esportivas específicas, intensidade e vigor de prática, persistência e continuidade para alcance de alto rendimento. Em vista disso, o presente estudo de revisão foi delineado para identificar os motivos associados à prática de esporte em jovens-atletas. Esta temática é revestida de grande importância para o campo do esporte, considerando que, suas indicações permitem identificar as principais razões que levam os jovens a iniciar a prática esportiva e que justificam sua permanência.

Análise dos artigos selecionados revelou que a Competência Técnica foi o principal motivo para prática de esporte apontado pelos jovensatletas de ambos os sexos inseridos nos diferentes contextos sociocultural considerados (Campos et al., 2011; Gill et al., 1983; Guedes \& Netto, 2013; Januário et al., 2012; Longhurst \& Spink, 1987; Paim, 2001). Este resultado está intimamente relacionado ao fato dos participantes estarem envolvidos em esportes de competição no âmbito escolar e de clubes esportivos. Os jovens-atletas consideram esse fator de motivação como determinante para sua permanência na equipe, privilegiam o aprimoramento técnico e buscam ser o melhor no esporte para alcançarem o sucesso. Tal comportamento deve-se ao fato de jovensatletas estarem preocupados com a aprendizagem e a execução de movimentos e gestos que envolvem cada modalidade esportiva específica. Desta maneira, jovens que têm melhor desempenho técnico são reconhecidos, enquanto aqueles menos habilidosos reforçam sentimentos de inabilidade. A importância dada ao fator Competência Técnica vai de encontro com convicções praticadas por muitos treinadores e gestores de clubes e escolas, em que o objetivo principal é a busca por resultados cada vez mais expressivos e vitórias.

Em contrapartida, estudos de Buonamano, Cei e Mussino (1995), Sit e Lindner (2006) e Garyfallos e Asterios (2011) identificaram que a Diversão como principal motivo tanto para rapazes como para moças. De fato, o esporte pode ser considerado atividade de entretenimento e divertimento, quando encarado de forma lúdica, em que as ações podem ser mais flexíveis e de menor rigor, e promovem um modo agradável de ocupar o tempo livre. A expressão diversão confirma que a participação de jovens-atletas no esporte é devido à necessidade de alegria e prazer, para liberar energia, sentir-se livre de preocupações e problemas do cotidiano (Garyfallos \& Asterios, 2011).

Nos achados de Balbinotti, Saldanha e Balbinotti (2009) e Balbinotti, Juchem, Barbosa, Saldanha e Balbinotti (2012) verificou-se o Prazer como sendo o fator de maior motivação independente do sexo. Prazer é considerado dimensão de destaque para compreensão dos 
motivos para prática de esporte. De acordo com Ryan e Deci (2000), o Prazer é a dimensão que melhor explica o comportamento humano autodeterminado, isso é, que melhor representa os motivos intrínsecos, inclusive no contexto esportivo. Neste caso, o Prazer provém unicamente da atividade em si, a participação ocorre livre de pressão e restrições, pode-se supor que atletas motivados pelo prazer ingressem no esporte por vontade própria, pela satisfação do processo de conhecê-lo, explorá-lo e aprofundá-lo.

Dos estudos analisados, alguns se concentraram no conhecimento quanto aos motivos do envolvimento de jovens-atletas na prática esportiva, independentemente de suas especificidades. A maioria dos estudos, no entanto, busca conhecer esta realidade não somente apontando um panorama generalizado, mas estratificando por sexo, idade, modalidades esportivas, tempo de prática e experiência de competição, a fim de determinar se os motivos que encorajam a prática de esporte são similares, ou pelo contrário, os argumentos se diferem. Neste caso, verificou-se que, sexo e idade foram os principais elementos diferenciadores dos motivos de prática esportiva em jovens-atletas.

No que se refere aos motivos relacionados ao sexo, os resultados indicaram que rapazes atribuíram grau de importância maior à Competição (Balbinotti et al., 2009; Curry \& Weiss, 1989; Guedes \& Netto, 2013; Januário et al., 2012; Kirkby et al., 1999), ao Reconhecimento Social (Gürbuz et al., 2007; Kirkby et al., 1999; Salguero et al., 2004; Sirard et al., 2006; Sit \& Lindner, 2006) e à Aptidão Física (Cecchini et al., 2002; Curry \& Weiss, 1989), sendo estes os principais motivos de permanência na prática de esporte. No entanto, nas moças verificou-se que os fatores Afiliação (Allen, 2003; Garyfallos \& Asterios, 2011; Guedes \& Netto, 2013; Kirkby et al., 1999; Salguero et al., 2004; Sirard et al., 2006; Sit \& Lindner, 2006) e Diversão (Allen, 2003; Balbinotti et al., 2009; Buonamano et al., 1995; Sit \& Lindner, 2006; Garyfallos \& Asterios, 2011; Kirkby et al.,
1999; Salguero et al., 2004; Salselas et al., 2007) justificaram em maior grau a prática de esporte. As moças se identificaram mais intensamente com motivos sociais e de convivência em grupo, o que indica seu desejo de identificar-se com seus pares e valorizar a importância nas relações pessoais na equipe em que esta inserida.

Buonamano et al. (1995) evidenciam que diversão na pratica esportiva esta intimamente relacionada às relações de amizade. Ressalta ainda que, para moças os motivos estão relacionados com o apoio externo oferecido por amigos, pais, treinadores e membros da equipe. Esses comportamentos, intrinsecamente motivados, são comumente associados à participação voluntária na prática de esporte, com aparente ausência de recompensas ou pressão externa, bem como participação no esporte pelo interesse, satisfação e alegria que obtêm de sua prática (Ryan \& Deci, 2000). Os rapazes, por sua vez, apresentaram fatores predominantemente extrínsecos, o que talvez possa ser justificado pelo fato dos rapazes utilizarem o esporte como forma de manifestação de aspectos relacionados à competição. Diferente das moças, os rapazes dispõem de mais oportunidades em esportes de alto nível, particularmente no âmbito profissional, buscando confronto, disputa, resultados, por consequência, comparação de desempenho consigo mesmo e com outros pode levá-los a se manter no esporte.

Quanto à idade, por vezes, os motivos relatados nos estudos não apresentaram coincidências de resultados, uma vez que, a classificação etária é configurada de acordo com categorias e características singulares da modalidade esportiva em que o atleta-jovem esta inserido. No entanto, buscou-se um denominador comum na analise dos artigos e constatou-se que, jovens-atletas com menos idade ( $\leq 14$ anos) atribuíram importância significativamente mais elevada à Diversão (Buonamano et al., 1995; Guedes \& Netto, 2013; Longhurst \& Spink, 1987; Martínez et al., 2008; Salguero et al., 2004), à Afiliação (Buonamano et al., 1995; Campos et al., 2011; Kirkby et al., 
1999; Longhurst \& Spink, 1987; Martínez et al., 2008; Paim, 2001; Salguero et al., 2004; Salselas et al., 2007) e ao Reconhecimentos Social (García et al., 2005; Guedes \& Netto, 2013; Martínez et al., 2008). Jovens-atletas com mais idade ( $\geq$ 15 anos), por sua vez, referem-se aos motivos equivalentes à Competição (Buonamano et al., 1995; Salguero et al., 2004; Salselas et al., 2007), à Competência Técnica (Paim, 2001; Salselas et al., 2007) e à Aptidão Fisica (Salselas et al., 2007) como os mais importantes para permanência na prática de esporte. Esses resultados confirmam tendência dos mais jovens em valorizar os componentes lúdicos e recreativos do esporte. Assim como, conferem importância para serem reconhecidos socialmente pela prática de esporte, ou seja, valorizam mais o receber elogios que receber medalhas e troféus, buscam obter reconhecimento de seus parentes e amigos e sentir-se importante e valorizado. De acordo com Martinez et al. (2008), a importância oferecida à Diversão tende a diminuir a medida que os jovens-atletas apresentam mais idade e, por consequência, mudam de categoria, conquistando, desse modo, maior independência paralelamente a aquisição de responsabilidades e deveres. Por essas razões, atletas com idades mais avançadas evidenciam motivos relacionados ao rendimento e à competência pessoal, rendendo significado mais competitivo à pratica esportiva.

Quanto ao âmbito de prática, Salselas et al. (2007) destacam que jovens-atletas de natação em nível competitivo evidenciaram Aptidão Física e Afiliação como motivos essenciais para a permanência na modalidade. Jovens-atletas em nível de competição, geralmente objetivam aprimorar suas habilidades e o suporte dos companheiros muitas vezes é fundamental no desenvolvimento da competência técnica. De acordo com Jõesaar e Hein (2011), jovensatletas com mais tempo de prática criam um senso de segurança e de convívio em grupo mais efetivo. Que pode, por sua vez, promover o encorajamento mútuo voltado ao aprimoramento de capacidades técnicas e tornar a atividade esportiva mais agradável, portanto, consolidar a permanência na pratica de esporte. Sugere-se, assim, que jovens-atletas mais experientes na prática de esporte e com maior envolvimento na modalidade podem oferecer importância tanto para motivos intrínsecos como extrínsecos.

Jovens-atletas iniciantes, por sua vez, atribuíram menor importância aos motivos equivalentes à Aptidão Física, à Competição, à Afiliação, à Competência Técnica e à Diversão quando comparados com seus pares de níveis avançados. Possivelmente, devido ao fato de jovens-atletas novatos sentirem-se hostilizados pelos companheiros de equipe mais experientes, gerando insegurança no domínio de suas habilidades e receio de cometer erros, tornando, portanto, a pratica esportiva insatisfatória e não-prazerosa (Salselas et al., 2007).

No que se refere à modalidade esportiva, a literatura disponibiliza limitada quantidade de estudos que procuram comparar os motivos que movem os jovens-atletas a optarem pela prática de esportes coletivo ou individual. De acordo com Guedes e Netto (2013), os jovens-atletas que praticavam esportes coletivos atribuíram considerável importância a Atividade em Grupo, a Competência Técnica e a Afiliação, enquanto os que praticavam esportes individuais valorizaram mais significativamente a Aptidão Física e a Competição. Em vista dos resultados apresentados, entende-se que a pratica de esportes coletivos por jovens-atletas esta associada à necessidade de aceitação e de pertencer a um grupo, sentimento importante na juventude. No esporte coletivo somam-se os valores individuais dos jovens-atletas, impera a cooperação, cada componente da equipe é dependente dos demais e atuam em conjunto para alcançar um objetivo comum. O esporte individual, no entanto, requer treinamento sistemático e competição regular contra outros atletas, demanda maior disciplina, concentração e introspecção, uma vez que os resultados alcançados dependem fundamentalmente do próprio atleta. 


\section{CONCLUSÕES}

Diante das considerações, pode-se concluir que, os motivos para a prática de esporte em jovens-atletas resultam da associação de diversos fatores sociais, ambientais e individuais. Os estudos selecionados para analise, em sua maioria, identificaram principalmente motivos vinculados à autorealização associada ao domínio/aperfeiçoamento de habilidades esportivas, em que a Competência Técnica foi o motivo para prática de esporte mais apontado pelos jovens-atletas de ambos os sexos inseridos nos diferentes contextos sociocultural. Identificou-se ainda, o sexo, a idade, o âmbito de pratica e a modalidade esportiva praticada como principais elementos diferenciadores dos motivos de prática esportiva em jovens-atletas.

Por fim, destaca-se que os estudos reunidos na presente revisão, na sua totalidade, se utilizaram de delineamentos transversais. Logo, a possível influência dos aspectos sociais, ambientais e individuais nos motivos para pratica de esporte deve ser considerada como um viés importante. Neste caso, evidencia-se a necessidade de futuros estudos relacionados a esta temática com delineamentos longitudinais, que permita acompanhamento dos motivos apontados ao longo do tempo, permitindo um entendimento mais efetivo quanto à eventual transição dos jovens-atletas pelos diferentes motivos selecionados. Assim, melhor compreender os motivos que podem contribuir para que os jovens iniciem e permaneçam na pratica de esporte e, ao contrário, as razões que os levam a abandonar a atividade esportiva.

\section{Agradecimentos:}

Nada a declarar

Conflito de Interesses:

Nada a declarar
Financiamento:

Nada a declarar

\section{REFERÊNCIAS}

Allen, J. (2003). Social motivation in youth Sports. Journal of Sport and Exercise Psychology, 25(4), 551-567.

Balbinotti, M. A. A., Juchem, L., Barbosa, M. L. L., Saldanha, R. P., \& Balbinotti, C. A. A. (2012). Qual é o perfil motivacional característico de tenistas infanto-juvenis brasileiros? Motriz, 18(4), 728-734.

Balbinotti, M. A. A., Saldanha, R. P., \& Balbinotti, C. A. A. (2009). Dimensões motivacionais de basquetebolistas infanto-juvenis: um estudo segundo o sexo. Motriz, 15(2), 318-329.

Boreham, C. A., Twisk, J., Savage, M. J., Cran, G. W., \& Strain, J. J. (1997). Physical activity, sports participation, and risk factors in adolescents. Medicine and Science in Sports and Exercise, 29(6), 788-793.

Buonamano, A., Cei, A., \& Mussino, A. (1995). Participation motivation in Italian Youth Sport. The Sports Psychology, 9(3), 265-281.

Campos, L. T. da S., Vigário, P. dos S., \& Lüdorf, S. M. A. (2011). Fatores motivacionais de jovens atletas de vôlei. Revista Brasileira de Ciências do Esporte, 33(2), 303-317. http://doi.org/10.1590/S010132892011000200003

Capranica, L., \& Millard-Stafford, M. L. (2011). Youth sport specialization: how to manage competition and training? International Journal of Sports Physiology and Performance, 6(4), 572-579.

Cecchini, J. A., Méndez, A., \& Muñiz, J. (2002). Motives for practicing sport in Spanish schoolchildren. Psicothema, 14(3), 523-531.

Curry, T. J., \& Weiss, O. (1989). Sport identity and motivation for sport participation: a comparison between American college athletes and Austrian student sport club members. Sociology of Sport Journal, 6(3), 257-268.

Delorme, N., Chalabaev, A., \& Raspaud, M. (2011). Relative age is associated with sport dropout: evidence from youth categories of French basketball. Scandinavian Journal of Medicine \& Science in Sports, 21(1), 120-128. http://doi.org/10.1111/j.16000838.2009.01060.x

García, F. G., Weis, G., \& Valdivieso, M. N. (2005). Motivos de participación deportiva de niños brasileños atendiendo a sus edades. Apunts: Educación Física y Deportes, 80, 29-36.

Garyfallos, A., \& Asterios, P. (2011). Motivation of 10-12 years old Cypriot students toward sports 
participation. Journal of Physical Education and Sport, 11(4), 401-405.

Gill, D. L., Gross, J. B., \& Huddleston, S. (1983). Participation motivation in youth sports. International Journal of Sports Psychology, 14, 1-14.

Gill, D. L., \& Williams, L. (2008). Psychological Dynamics of Sport and Exercise (3rd ed.). Champaign, Illinois: Human Kinetics.

Guedes, D. P., \& Netto, J. E. S. (2013). Sport participation motives of young Brazilian athletes. Perceptual and Motor Skills, 117(3), 742-759. http://doi.org/10.2466/06.30.PMS.117x33z2

Gürbuz, B., Altyntas, A., Asci, F., \& Hülya, A. (2007). Participation motives of 9-15 years old Turkish soccer players. Journal of Sports Science and Medicine, 7(10), 89-90.

Harrison, P. A., \& Narayan, G. (2003). Differences in behavior, psychological factors, and environmental factors associated with participation in school sports and other activities in adolescence. The Journal of School Health, 73(3), 113-120.

Higgins, J. P. T., \& Green, S. (Eds.). (2008). Cochrane Handbook for Systematic Reviews of Interventions. Chichester, UK: John Wiley \& Sons, Inc.

Interdonato, C. G., Miarka, B., Oliveira, A. R., \& Gorgatti, M. G. (2008). Fatores motivacionais de atletas para a prática esportiva. Motriz, 14(1), 63-66.

Januário, N., Colaço, C., Rosado, A., Ferreira, V., \& Gil, R. (2012). Motivação para a prática desportiva nos alunos do ensino básico e secundário: Influência do género, idade e nível de escolaridade. Motricidade, 8(4), 38-51. http://doi.org/10.6063/motricidade.8(4).1551

Jõesaar, H., \& Hein, V. (2011). Psychosocial determinants of young athletes' continued participation over time. Perceptual and Motor Skills, 113(1), 51-66.

http://doi.org/10.2466/05.06.13.PMS.113.4.5 $1-66$

Kawabe, H., Murata, K., Shibata, H., Hirose, H., Tsujioka, M., Saito, I., \& Saruta, T. (2000). Participation in school sports clubs and related effects on cardiovascular risk factors in young males. Hypertension Research, 23(3), 227-232.

Kirkby, R. J., Kolt, G. S., \& Liu, J. (1999). Participation motives of young Australian and Chinese gymnasts. Perceptual and Motor Skills, 88(2), 363-373.

http://doi.org/10.2466/pms.1999.88.2.363

Longhurst, K., \& Spink, K. S. (1987). Participation motivation of Australian children involved in organized sport. Canadian Journal of Sport Sciences = Journal Canadien Des Sciences Du Sport, 12(1), 24-30.

Lopes, P., \& Nunomura, M. (2007). Motivação para a prática e permanência na ginástica artística de alto nível. Revista Brasileira de Educação Física e Esporte, 21(3), 177-187.

Martínez, R., Molinero, O., Jiménez, R., Salguero, A., Tuero, C., \& Márquez, S. (2008). La motivación para la práctica en la iniciación al fútbol: influencia de la edad/categoría competitiva, el tiempo de entrenamiento y la relación con el entrenador. Apunts: Educación Física y Deportes, 93(3), 46-54.

Nuñez, P. R. M., Picada, H. F. S. de L., Schulz, S. T., Habitante, C. A., \& da Silva, J. V. P. (2008). Motivos que levam adolescentes a praticar o futsal. Conexões, 6(1), 67-78.

Paim, M. C. C. (2001). Fatores motivacionais e desempenho no futebol. Revista da Educação Física/UEM, 12(2), 73-79. http://doi.org/10.4025/reveducfisv12n2p7379

Paim, M. C. C., \& Pereira, E. (2004). Fatores motivacionais dos adolescentes para a prática de capoeira na escola. Motriz, 10(3), 159-166.

Pate, R. R., Trost, S. G., Levin, S., \& Dowda, M. (2000). Sports participation and health-related behaviors among US youth. Archives of Pediatrics \& Adolescent Medicine, 154(9), 904-911.

Roberts, G. C., \& Treasure, D. C. (2012). Advances in Motivation in Sport and Exercise-3rd Edition (3rd ed.). Champaign, Illinois: Human Kinetics.

Ryan, R. M., \& Deci, E. L. (2000). Self-determination theory and the facilitation of intrinsic motivation, social development, and well-being. American Psychologist, 55(1), 68-78. http://doi.org/10.1037/0003-066X.55.1.68

Salguero, A., Gonzalez-Boto, R., Tuero, C., \& Marquez, S. (2004). Relationship between perceived physical ability and sport participation motives in young competitive swimmers. The Journal of Sports Medicine and Physical Fitness, 44(3), 294-299.

Salselas, V., Gonzalez-Boto, R., Tuero, C., \& Marquez, S. (2007). The relationship between sources of motivation and level of practice in young Portuguese swimmers. The Journal of Sports Medicine and Physical Fitness, 47(2), 228-233.

Sirard, J. R., Pfeiffer, K. A., \& Pate, R. R. (2006). Motivational factors associated with sports program participation in middle school students. The Journal of Adolescent Health: Official Publication of the Society for Adolescent Medicine, 38(6), 696-703. http://doi.org/10.1016/j.jadohealth.2005.07.0 13

Sit, C. H. P., \& Lindner, K. J. (2006). Situational state balances and participation motivation in youth sport: a reversal theory perspective. The British Journal of Educational Psychology, 76( $\mathrm{Pt} 2)$, 369-384.

http://doi.org/10.1348/000709905X37190 
Weinberg, R., Tenenbaum, G., McKenzie, A., Jackson, S., Anshel, M., Grove, R., \& Fogarty, G. (2000). Motivation for youth participation in sport and physical activity: relationships to culture, self-reported activity levels, and gender. International Journal of Sport Psychology, 31, 321-346.
Zanetti, M. C., Lavoura, T. N., \& Machado, A. A. (2008). Motivação no esporte infanto- juvenil. Conexões, 6(0). Obtido de http://fefnet178.fef.unicamp.br/ojs/index.php/fef/article/view/264

Todo o conteúdo da revista Motricidade está licenciado sob a Creative Commons, exceto quando especificado em contrário e nos conteúdos retirados de outras fontes bibliográficas. 